\title{
Plasminogen activator inhibitor-1, free fatty acids, and insulin resistance in patients with myocardial infarction
}

This article was published in the following Dove Press journal:

Diabetes, Metabolic Syndrome and Obesity:Targets and Therapy

12 August 2013

Number of times this article has been viewed

\section{Olga Gruzdeva \\ Evgenya Uchasova \\ Yulia Dyleva \\ Ekaterina Belik \\ Ekaterina Shurygina \\ Olga Barbarash}

Research Institute for Complex Issues of Cardiovascular Diseases under the Siberian Branch of the Russian Academy of Medical Sciences, Kemerovo, Russian Federation

Correspondence: EG Uchasova FSBI Research Institute for Complex Issues of Cardiovascular Diseases SB RAMS, 6 Sosnovy Bvld, Kemerovo 650002, Russian Federation

Tel +7 3842640553

Email evg.uchasova@yandex.ru
Background: Insulin resistance is known to be a common feature of type 2 diabetes mellitus and is regarded as an important mechanism in the pathogenesis of this disease. The key pathogenetic mechanisms of insulin resistance progression are free fatty acids metabolism impairment and enhanced activity of plasminogen activator inhibitor 1 . Both free fatty acids and plasminogen activator inhibitor 1 are recognized as risk factors for coronary heart disease.

Methods: The patients were divided into two groups: group 1 included 65 non-diabetic myocardial infarction patients and group 2 enrolled 60 diabetic myocardial infarction patients. The control group consisted of 30 sex- and age-matched volunteers. The concentration of serum free fatty acids, glucose, C-peptide, and insulin were measured on the 1st and 12th days of the study. All the patients had their postprandial glycemia, insulin, and C-peptide concentrations measured 2 hours after a standard carbohydrate breakfast containing $360 \mathrm{kcal}$ (protein $20 \mathrm{~g}$, carbohydrate $57 \mathrm{~g}$, and fat $9 \mathrm{~g}$ ).

Results: Free fatty acids levels in group 1 and in group 2 exceeded the control group values by 7-fold and 11-fold, respectively. Plasminogen activator inhibitor 1 concentration was 2.5-fold higher in group 1 and 4.6-fold higher in group 2 compared to the control group on the 1st day from the myocardial infarction onset. In addition, plasminogen activator inhibitor 1 concentration was significantly reduced in both groups on the 12th day from the myocardial infarction onset; however, it did not achieve the control group values.

Conclusion: Increased postprandial glucose level, insulinemia, and elevated levels of free fatty acids and plasminogen activator inhibitor are associated with myocardial infarction-associated progression of insulin resistance. However, insulin resistance metabolic markers are of great predictive capacity in the assessment of risk of acute coronary events.

Keywords: free fatty acids, type 2 diabetes mellitus, myocardial infarction, insulin resistance, plasminogen activator inhibitor 1

\section{Introduction}

Coronary heart disease (CHD) and myocardial infarction (MI) have a significant impact on morbidity and mortality in developed countries. ${ }^{1,2}$ Thrombosis induced by atherosclerotic plaques rupture and subsequent distal thromboembolism may result in MI progression. ${ }^{1,3}$ Thrombus formation is regulated by the fibrinolytic system, which prevents luminal occlusion. Predominance of fibrinolysis inhibitors over its activators and plasminogen deficiency may be considered as risk factors for atherosclerosis and MI. Plasminogen activator inhibitor 1 (PAI-1) plays a significant role in cardiovascular diseases. Clinical studies have shown a positive correlation between increased levels of PAI-1 and atherothrombosis. ${ }^{3}$ Moreover, high plasma PAI-1 concentration is considered to be a predictor of myocardial infarction. ${ }^{1}$ PAI- 1 is involved in the insulin reception process as 
well as in fibrinolysis regulation. PAI-1 also modulates insulin signaling in fibroblasts, preventing the binding of vitronectin to avb3 receptors that, in turn, reduces insulin-induced phosphorylation of protein kinase B. ${ }^{4,5}$ A positive correlation between plasma PAI-1 concentration and insulin resistance (IR) markers was verified by epidemiological studies. ${ }^{6}$

According to various theories, IR is considered a common feature of type 2 diabetes mellitus (T2DM) and is regarded as an important mechanism in the pathogenesis of this disease. Cardiovascular risk factors, including hyperglycemia, dyslipoproteinemia, hypertension, obesity, thrombosis, and smoking are also associated with increased IR risk. ${ }^{6}$ Nowadays, the results of several studies have already been published confirming the association of IR with atherosclerosis manifestations and cardiovascular risk in both men and women. ${ }^{7}$

Impaired free fatty acid (FFA) metabolism and increased blood FFA levels together with the impaired glucose utilization are significant pathogenetic mechanisms of the IR progression. Increased FFA levels are considered to be an early IR marker, which can be revealed long before glucose intolerance and T2DM progression. ${ }^{8}$ FFA is traditionally considered as a main myocardium metabolic resource. ${ }^{9}$ Intensity of FFA transport to the myocardial tissues is determined by their plasma concentration. Anaerobic glycolysis is supposed to be a main metabolic pathway under ischemic conditions by providing energy to cardiomyocytes, since FFA oxidation is associated with higher oxygen consumption. This phenomenon may lead to FFA utilization and decrease of blood FFA levels. ${ }^{10}$ Moreover, disruption of mitochondrial respiratory enzymes under hypoxic conditions results in oxidative modification of lipoproteins, induces endothelial inflammation, and promotes atherosclerotic plaques formation and ischemia progression. ${ }^{9,11}$ Reversible metabolic impairment in the early stages becomes inevitably irreversible and leads to cell death in the absence of reperfusion. ${ }^{13,14}$ Thus, literature analysis suggests PAI-1 and FFA involvement in IR progression, which is recognized as a CHD risk factor. The determination of MI progressionrelated IR markers is of great importance for the assessment of further treatment and prognosis.

Therefore, the aim of this study is to access insulin resistance marker dynamics in ST-segment elevation patients with myocardial infarction with presented and non-presented T2DM in acute and post-acute rehabilitation periods.

\section{Material and methods \\ Study subjects and design}

One-hundred and twenty-five MI patients (65 males and 60 females), mean age \pm standard deviation $65 \pm 4.5$ years, and 30 sex and age-matched volunteers (the control group) with no cardiovascular or endocrine diseases were enrolled in the study. The patients were divided into two groups: group 1 included 65 non-diabetic MI patients and group 2 enrolled 60 diabetic MI patients. The mean \pm standard deviation T2DM duration was, on average, $6.4 \pm 1.5$ years. The groups were sex and age-matched and had similar risk

Table I Initial clinical and anamnestic characteristics of patients, $\mathrm{n}(\%)$

\begin{tabular}{|c|c|c|c|}
\hline Variable & $\begin{array}{l}\text { Patients with MI without type } 2 \\
\text { diabetes }(n=65)\end{array}$ & $\begin{array}{l}\text { Patients with MI and type } 2 \\
\text { diabetes }(n=60)\end{array}$ & $\begin{array}{l}\text { Control } \\
(n=30)\end{array}$ \\
\hline Men, n (\%) & $44(73.3)$ & $18(30)$ & $18(60)$ \\
\hline Age, year & $61(53 ; 70)$ & $66,5(6 I ; 73,5)$ & $58(51 ; 62)$ \\
\hline Body mass index, $\mathrm{kg} / \mathrm{m}^{2}$ & $25.5(23.2 ; 30.1)$ & $29.65(26.85 ; 34.55)$ & $23.6(21.3 ; 26.5)$ \\
\hline CK MB, U/L & $137.64(99.5 ; 165,1)$ & $94.03(80,3 ; 110,3)$ & $15.2(11,3 ; 19,3)$ \\
\hline Troponin T, ng/mL & $0.71(0.36 ; 1.02)$ & $1.09(0,95 ; 1,16)$ & $0.09(0.08 ; 0.12)$ \\
\hline Overweight, n (\%) & $37(56.9)$ & $51(85)$ & \\
\hline Arterial hypertension, n (\%) & $50(79.6)$ & $51(85)$ & \\
\hline Hypercholesterolemia, n (\%) & $25(38.5)$ & $27(45)$ & \\
\hline Current smoking, n (\%) & $50(79.6)$ & $37(6 \mid .6)$ & $2(6.6)$ \\
\hline Family history of IHD, n (\%) & $16(24.6)$ & $30(50)$ & $5(16)$ \\
\hline Previous myocardial infarction, $\mathrm{n}(\%)$ & $10(15.4)$ & $15(25)$ & \\
\hline Ulcerous disease, n (\%) & $6(9.3)$ & $5(8.3)$ & \\
\hline Chronic kidney disease, n (\%) & $\mathrm{I}(2.1)$ & I (I.6) & \\
\hline Anterior wall myocardial infarction, $\mathrm{n}$ (\%) & $45(42)$ & $9(15)$ & \\
\hline Killip class I, n (\%) & $53(81.5)$ & $26(43.3)$ & \\
\hline Killip class II-IV, n (\%) & $21(35.4)$ & $32(53.3)$ & \\
\hline Death, n (\%) & I (0.9) & I (I.6) & \\
\hline
\end{tabular}

Notes: Age and body mass index and are presented as median and quartiles $\left(M e\left[Q_{1} ; Q_{3}\right]\right)$.

Abbreviations: CK MB, myocardial fraction of creatine phosphokinase; IHD, ischemic heart disease; MI, myocardial infaraction. 
factors for ischemic heart disease, concurrent conditions, and MI complications rate.

Main demographic characteristics of the study patients and group control are summarized in Table 1. The patient groups were similar in age, sex main risk factors for ischemic heart disease, comorbidities, and coronary events incidence (Table 1).

Acute myocardial infarction was diagnosed according to the 2007 Russian National Cardiology Society guidelines based on clinical, electrocardiographic (ECG), echocardiographic (ECHO), and biochemical signs of the disease. The inclusion criteria were chest pain refractory to nitroglycerin, myocardial ischemia, and signs of necrosis (ST segment elevation and/or new pathologic Q waves on the ECG, elevated cardiac enzymes, myocardial fraction of creatine phosphokinase [CK MB], and troponin T). Peak CK MB and troponin $\mathrm{T}$ levels did not differ between groups (Table 1). Peak CK MB levels were $94.03 \pm 17.9 \mathrm{U} / \mathrm{L}$ and $137.64 \pm 41.1 \mathrm{U} / \mathrm{L}$ in the diabetic and non-diabetic patients, respectively $(P=0.916)$; troponin $\mathrm{T}$ concentrations were $1.09 \pm 0.92 \mathrm{ng} / \mathrm{mL}$ and $0.71 \pm 0.41 \mathrm{ng} / \mathrm{mL}$ in the diabetic and non-diabetic patients, respectively $(P=0.564)$. In addition, all patients had their C-reactive protein (CRP) levels measured; concentrations were $20.47(9.59 ; 45.85) \mathrm{mg} / \mathrm{L}$ and 29.86 $(27.3 ; 31.9) \mathrm{mg} / \mathrm{L}$ (presented as median and quartiles $[\mathrm{Me}$ $\left.\left.\left\{\mathrm{Q}_{1} ; \mathrm{Q}_{3}\right\}\right]\right)$ in the diabetic and non-diabetic patients, respectively $(P=0.001)$. The exclusion criteria were age $>75$ years, clinically significant comorbidities (autoimmune diseases, thyroid, or adrenal gland diseases), acute coronary syndrome as a complication of percutaneous coronary intervention, or coronary artery bypass surgery. Exclusion criteria for patients with MI without diabetes, was as well, the presence of glucose on an empty stomach of more than $7 \mathrm{mmol} / \mathrm{L}$.

Q-wave MI was diagnosed in 18 diabetic and 20 nondiabetic patients $(P=0.866)$. The patient groups had a similar incidence of major hospital coronary events (Table 1). In both groups, $80 \%$ of patients did not have any signs of acute heart failure, according to the 1967 Killip classification upon admission and during the hospital stay (Table 1); however, more than $50 \%$ of patients had arrhythmias and impaired conduction diagnosed according to established criteria. ${ }^{12}$ The structure and function of the left ventricle did not differ in the groups. Mean left ventricular ejection fraction was $51.25 \% \pm 1.85 \%$ in the diabetic patients and $50.42 \% \pm 1.62 \%$ in those without T2DM $(P=0.598)$; mean left ventricular enddiastolic volume was $164.35 \pm 10.47 \mathrm{~mL}$ in the diabetic patients and $156.43 \pm 7.18 \mathrm{~mL}$ in the non-diabetic patients
$(P=0.649)$; mean left ventricular end-systolic volume was $79.71 \pm 8.32 \mathrm{~mL}$ in the diabetic patients and $75.22 \pm 5.56 \mathrm{in}$ the non-diabetic patients $(P=0.702)$.

The groups were similar in the presence/type of coronary artery disease: coronary angiography showed multivessel ( $>3$ vessels) hemodynamically significant coronary artery lesions in both groups $(76.5 \%$ in the diabetic patients and $62.5 \%$ in the non-diabetic patients; $P=0.857$ ).

Primary percutaneous coronary intervention (PCI) of the infarct-related artery was used as reperfusion therapy and, if contraindicated (PCI technically unfeasible), systemic thrombolysis with streptokinase (1.5 million units) or pharmacological treatment was administered. PCI frequency was similar in both groups: it was done for 16 diabetic patients and 24 non-diabetic patients $(P=0.512)$. All patients received medication therapy, if not contraindicated, including acidum acetylsalicylicum (ASA), clopidogrel angiotensin converting enzyme (ACE), inhibitors and antianginal drugs; all medications were administered according to the standard of care. Fourteen diabetic patients took antihyperglycemic medications before MI: six patients took sulfonylurea drugs, four patients biguanides, three patients took a combination of sulfonylurea drugs and biguanides, and one patient took dipeptidyl peptidase-4 (DPP-4) inhibitors in combination with biguanides. Six patients were treated with the diet only. No patients showed any bleeding as a consequence of the therapy.

T2DM compensation was evaluated based on glycated hemoglobin $\mathrm{HbA}_{1 \mathrm{c}}$ levels (T2DM was considered to be compensated in $\mathrm{HbA}_{1 \mathrm{c}}>7.5 \%$, according to the 2000 European Diabetes Policy Group criteria). Decompensated T2DM was diagnosed in eight patients.

The study design was approved by the Institutional Review Board of the Research Institute for Complex Issues of Cardiovascular Diseases; all individuals signed informed consent before being enrolled in the study. Therapeutic interventions during the follow-up period are listed in Table 2.

Table 2 Revascularization and drug therapy during follow-up

\begin{tabular}{lll}
\hline Therapy, $\mathbf{n}(\%)$ & & \\
\hline B-blockers & $96(89.7)$ & $55(84.6)$ \\
Angiotensin-converting enzyme & $88(82.2)$ & $51(85)$ \\
Calcium channel blocker & $75(70)$ & $21(65.6)$ \\
Diuretics & $45(42)$ & $39(60)$ \\
Nitrates & $94(87.8)$ & $31(51.6)$ \\
Aspirin & $94(87.8)$ & $55(91.6)$ \\
Heparin & $95(100)$ & $60(100)$ \\
Clopidogrel & $84(78.5)$ & $44(73.3)$ \\
Statins & $95(100)$ & $60(100)$ \\
\hline
\end{tabular}




\section{Assay}

Blood serum and plasma were tested. The serum was separated from venous blood by centrifugation at $3000 \mathrm{~g}$ for 20 minutes and stored at $-70^{\circ} \mathrm{C}$. The tests were done on the 1 st day from MI and acute heart failure onset and then on the 12th day, after the condition had been stabilized. FFA and glucose concentrations as biochemical markers of IR were measured at the same study time points using standard Thermo Fisher Scientific test systems (Thermo Fisher Scientific Oy, Vantaa, Finland) on a Konelab 30i biochemistry analyzer (Thermo Fisher Scientific Oy). C-peptide and insulin concentrations were measured by enzyme-linked immunosorbent assay (ELISA) with BioMedica (Waterloo, NSW, Australia) and Diagnostic Systems Laboratories (Webster, TX, USA) lab kits, respectively. The intra-assay coefficients of variation (CVs) for insulin Diagnostic Systems Laboratories and C-peptide ELISA were $3.8 \%$ and $4.2 \%$, respectively, and the inter-assay CVs were $6.9 \%$ and $7.9 \%$, respectively. Additionally, all patients had their postprandial glycemia, insulin, and C-peptide concentrations measured 2 hours after being fed a standard solid meal containing $360 \mathrm{kcal}$ (protein $20 \mathrm{~g}$, carbohydrate $57 \mathrm{~g}$, and fat $9 \mathrm{~g}$ ).

IR levels were estimated using a structural mathematical model incorporating fasting serum insulin and glucose measurement with the Quantitative Insulin Sensitivity Check Index (QUICKI) calculation:

$$
\text { QUICKI }=1 /[\log (\mathrm{I} 0)+\log (\mathrm{G} 0)]
$$

where I0 is basal insulinemia $(\mathrm{mg} / \mathrm{dL})$ and $\mathrm{G} 0$ is basal glycemia $(\mathrm{mME} / \mathrm{mL}) .{ }^{15}$ The average QUICKI value of $0.382+0.007$ corresponds to normal tissue sensitivity to insulin; the QUICKI value of $0.331+0.010$ and $0.304+0.007$ corresponds to moderate and severe tissue IR, respectively.

\section{Statistical analysis}

Statistical analysis was done using non-parametric MannWhitney test for unpaired samples and Wilcoxon test for paired samples. The results were presented as median and quartiles $\left(\mathrm{Me}\left[\mathrm{Q}_{1} ; \mathrm{Q}_{3}\right]\right) . P$ values $<0.05$ were considered statistically significant. Spearman's correlation coefficient was calculated to analyze correlations between the variables. Statistical analysis was performed using Statistica 6.0 (StatSoft, Tulsa, OK, USA).

\section{Results}

Blood glucose levels increased 1.2-fold in group 1 and 1.9-fold in group 2 compared to the control group on the 1st day after MI onset (Table 3). An elevation in glucose level

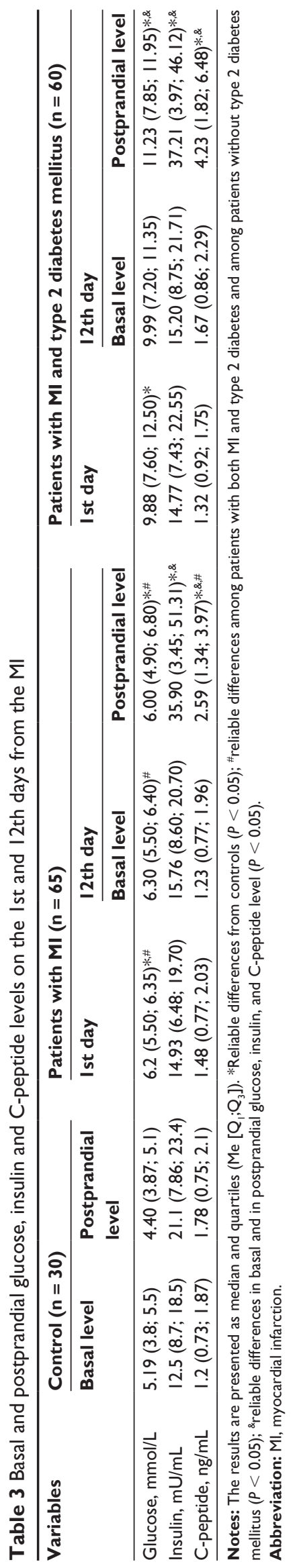


was noticed in group 2 during the entire follow-up period, whereas in group 1, blood glucose levels decreased on the 12th day from MI onset but did not achieve the values in the control group. In addition, postprandial glucose levels increased 2.6-fold in group 2 in comparison to the control values on the 12th day from the MI onset (Table 3 ).

In contrast, fasting serum insulin and C-peptide levels tended to increase in both groups on the 1 st and 12 th days from MI onset, but did not differ significantly from the control values (Table 3). The changes in postprandial values were more significant on the 12th day of MI progression (Table 3). Postprandial insulin level raised equally in both groups compared to the control group. The change in C-peptide dynamics was comparable with the change in glucose level (C-peptide concentration increased 2.3-fold in group 2 and 1.5-fold in group 1).

The IR QUICKI integral index significantly differed from the control values in both groups despite the fact that carbohydrate metabolism changes were less pronounced in group 1 than in group 2. The QUICKI index in the control group was $0.38 \pm 0.01$ and corresponded to normal tissue sensitivity to insulin. In group 1, the QUICKI indices were $0.316 \pm 0.005$ and $0.319 \pm 0.005$ on the 1 st and 12 th days after the MI onset, respectively, and they were interpreted as breakpoint values between severe and moderate IR. In group 2, the QUICKI indices were $0.296 \pm 0.009$ and $0.300 \pm 0.005$ that, in accordance with Katz et al, verified pronounced tissue IR. ${ }^{15}$

Significant differences in FFA concentration were detected in patients with MI on the 1st and 12th days from the MI onset compared to healthy subjects (Table 4). FFA levels in group 1 and in group 2 exceeded the control group values by 7 -fold and 11-fold, respectively. On the 12th day of follow-up they decreased, but still differed by 3.0-4.7-fold from the normal values. The changes were more remarkable in group 2 .

PAI-1 concentration was 2.5-fold higher in group 1 and 4.6-fold higher in group 2 compared to the control group on the 1 st day from MI onset. In addition, PAI-1 concentration significantly reduced in both groups on the 12th day from MI onset; however, it did not achieve control group values (Table 4).
Postprandial insulin positively correlated with FFA levels $(R=0.395, P=0.0002)$ (Figure 1$)$ and QUICKI index negatively correlated with FFA levels $(R=-0.74, P=0.0002$; Figure 2) in group 1. Moreover, a negative correlation between PAI-1 concentration and QUICKI index was reported in group 2 and in group $1(R=-0.77, P=0.005 ; R=-0.47, P=0.005$, respectively; Figure 3); PAI-1 and FFA levels were positively correlated $(R=0.235, P=0.0088$; Figure 4$)$.

\section{Discussion}

According to the modern point of view, IR is considered a key pathogenetic mechanism of T2DM, and it is also regarded as an atherosclerosis risk factor together with the metabolic markers, including serum glucose and insulin levels. ${ }^{9}$ The results of our study indicate the presence of basal and postprandial hyperglycemia in patients with MI in acute and post-acute rehabilitation periods; it was also found that blood glucose levels are higher in MI patients with associated T2DM during the follow-up period. The presence of basal and postprandial hyperglycemia in MI patients was considered to be an independent risk factor for adverse events according to the results of clinical studies. ${ }^{2}$ The authors noted a high number of in-hospital complications and high case fatality rate in patients with $\mathrm{MI}$ and associated hyperglycemia during a long-term period. According to several studies, postprandial insulin and C-peptide levels are more informative than basal levels, particularly in cases without obvious IR manifestations, and the detected analyte can be used for IR identification. ${ }^{6}$ However, postprandial insulin and C-peptide levels were significantly higher in both case groups compared to healthy subjects, but only slightly differed within the case groups. The presence of IR in patients included in the study was confirmed by the low QUICKI index. The severe IR was reported in the case of combined pathology, and moderate IR degree was reported in cases with diabetes absence.

Reduced sensitivity to insulin also affects lipid metabolism; initially FFA metabolism. Although we found a statistically significant increase in FFA levels in both groups of patients who suffered from acute MI, the results were more

Table 4 Basal level of FFA and PAI-1, on the Ist and I2th days from the MI, respectively

\begin{tabular}{|c|c|c|c|c|c|}
\hline \multirow[t]{2}{*}{ Variables } & \multirow[t]{2}{*}{$\begin{array}{l}\text { Control } \\
(n=30)\end{array}$} & \multicolumn{2}{|c|}{ Patients with MI $(n=65)$} & \multicolumn{2}{|c|}{$\begin{array}{l}\text { Patients with MI and type } 2 \text { diabetes mellitus } \\
(n=60)\end{array}$} \\
\hline & & Ist day & 12th day & Ist day & I2th day \\
\hline $\mathrm{FFA}, \mathrm{mmol} / \mathrm{L}$ & $0.20(0.1 ; 1.1)$ & $\mathrm{I} .4 \mathrm{I}(0.93 ; 1.92)^{* \# \#}$ & $0.6 \mathrm{I}(0.4 \mathrm{I} ; 0.87)^{* *, \#}$ & $2.20(1.47 ; 2.78)^{*}$ & $0.93(0.68 ; 1.28)^{* *}$ \\
\hline PA-I, ng/mL & $35.25(28.3 ; 46.3)$ & $86.44(64.36 ; 136.20)^{*, \#}$ & $71.21(37.61 ; 102.10)^{* * . \#}$ & $161.09(131.15 ; 177.00)^{*}$ & $107.64(74.8 \mathrm{I} ; 166.40)^{* *}$ \\
\hline
\end{tabular}

Notes: The results are presented as median and quartiles $\left(M e\left[Q_{1} ; Q_{3}\right]\right)$. *Reliable differences from controls, $(P<0.05)$; **reliable differences in the indicators on the Ist and 12th day $(P<0.05)$; ${ }^{*}$ reliable differences among patients with both $\mathrm{Ml}$ and type 2 diabetes and among patients without type 2 diabetes mellitus $(P<0.05)$.

Abbreviations: FFA, free fatty acid; MI, myocardial infarction; PAI-1, plasminogen activator inhibitor-1. 
FFA, $\mathrm{mmol} / \mathrm{L}=1+0 * x$

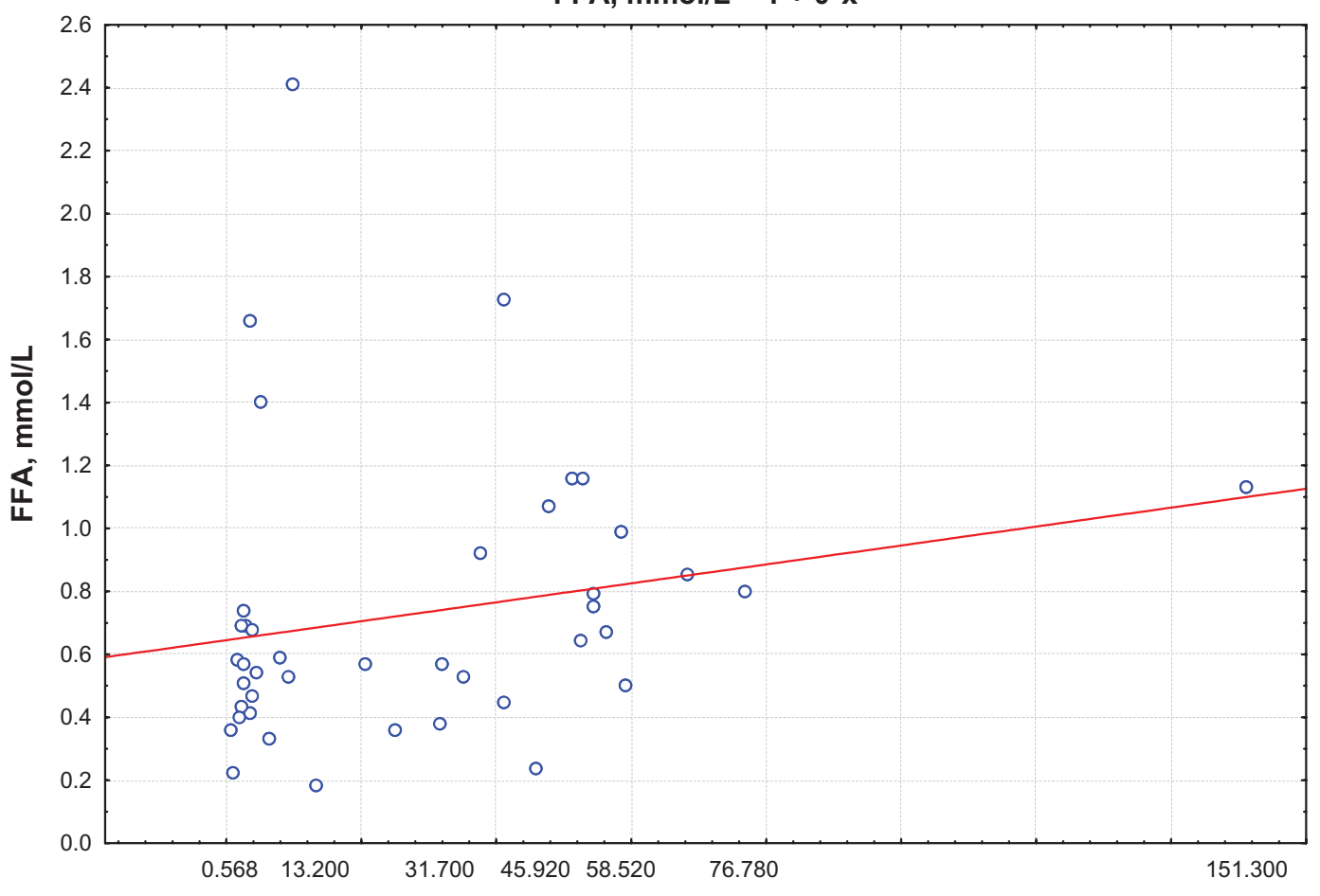

Postprandial insulin, $\mathrm{mU} / \mathrm{mL}$

Figure I Correlation between postprandial insulin and FFA levels.

Abbreviation: FFA, free fatty acid.

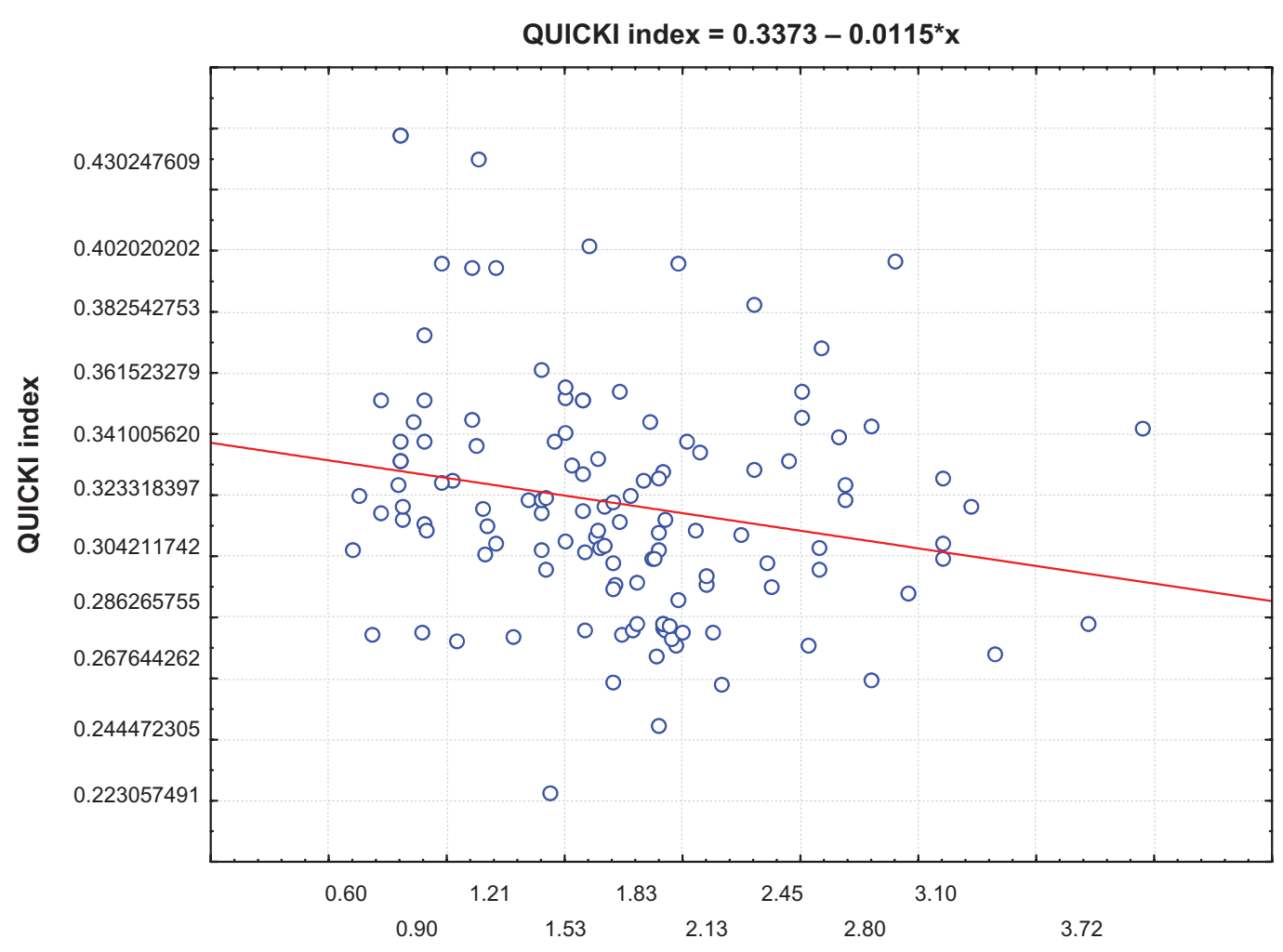

FFA, $\mathrm{mmol} / \mathrm{L}$

Figure 2 Correlation between QUICKI index and FFA levels.

Abbreviations: FFA, free fatty acid; QUICKI, Quantitative Insulin Sensitivity Check Index. 


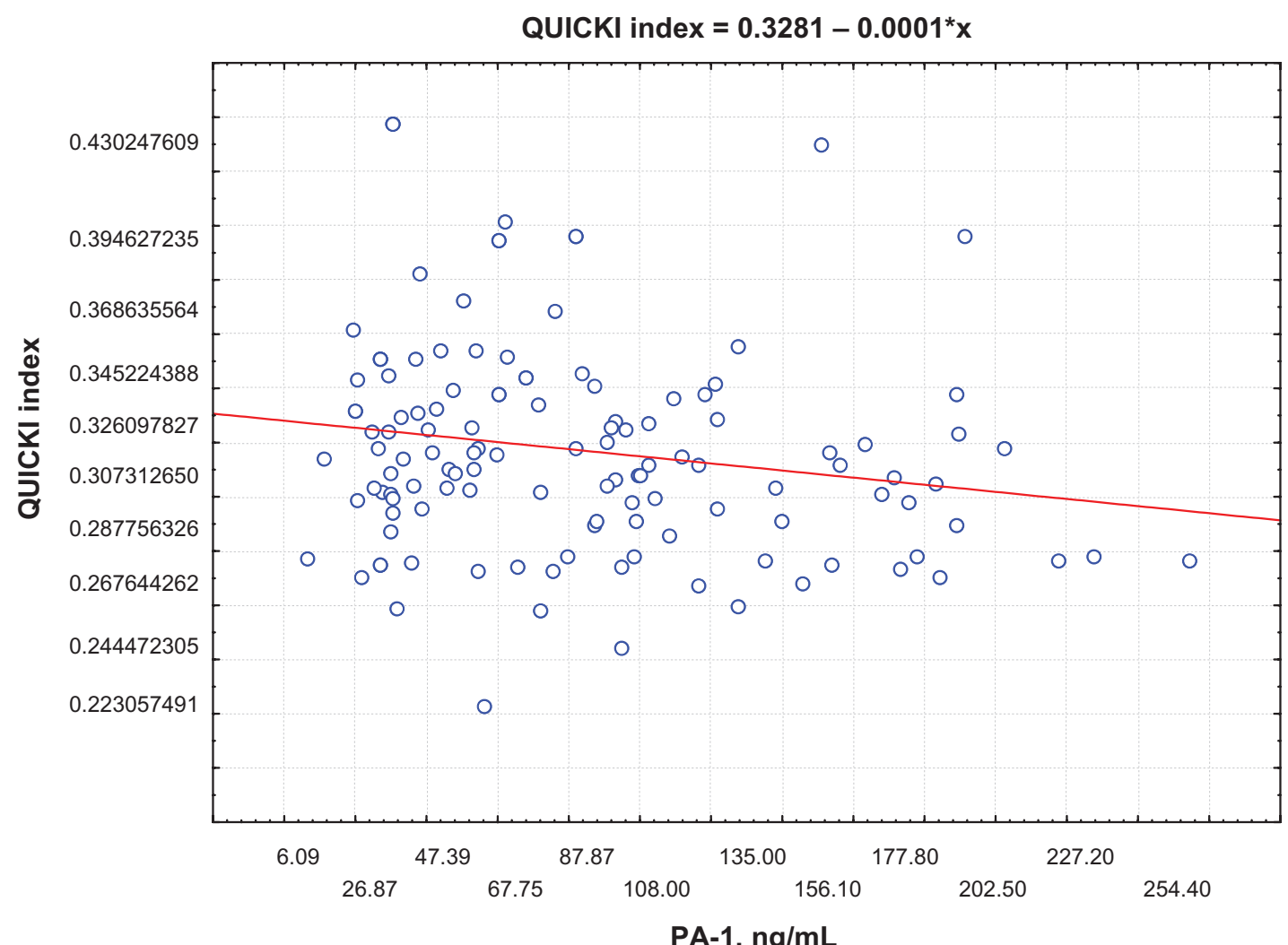

Figure 3 Correlation between QUICKI index and PAI levels.

Abbreviations: PAI, plasminogen activator inhibitor; QUICKI, Quantitative Insulin Sensitivity Check Index.

$\mathrm{PAl}-1, \mathrm{ng} / \mathrm{mL}=58.2691+37.6042^{*} \mathrm{X}$

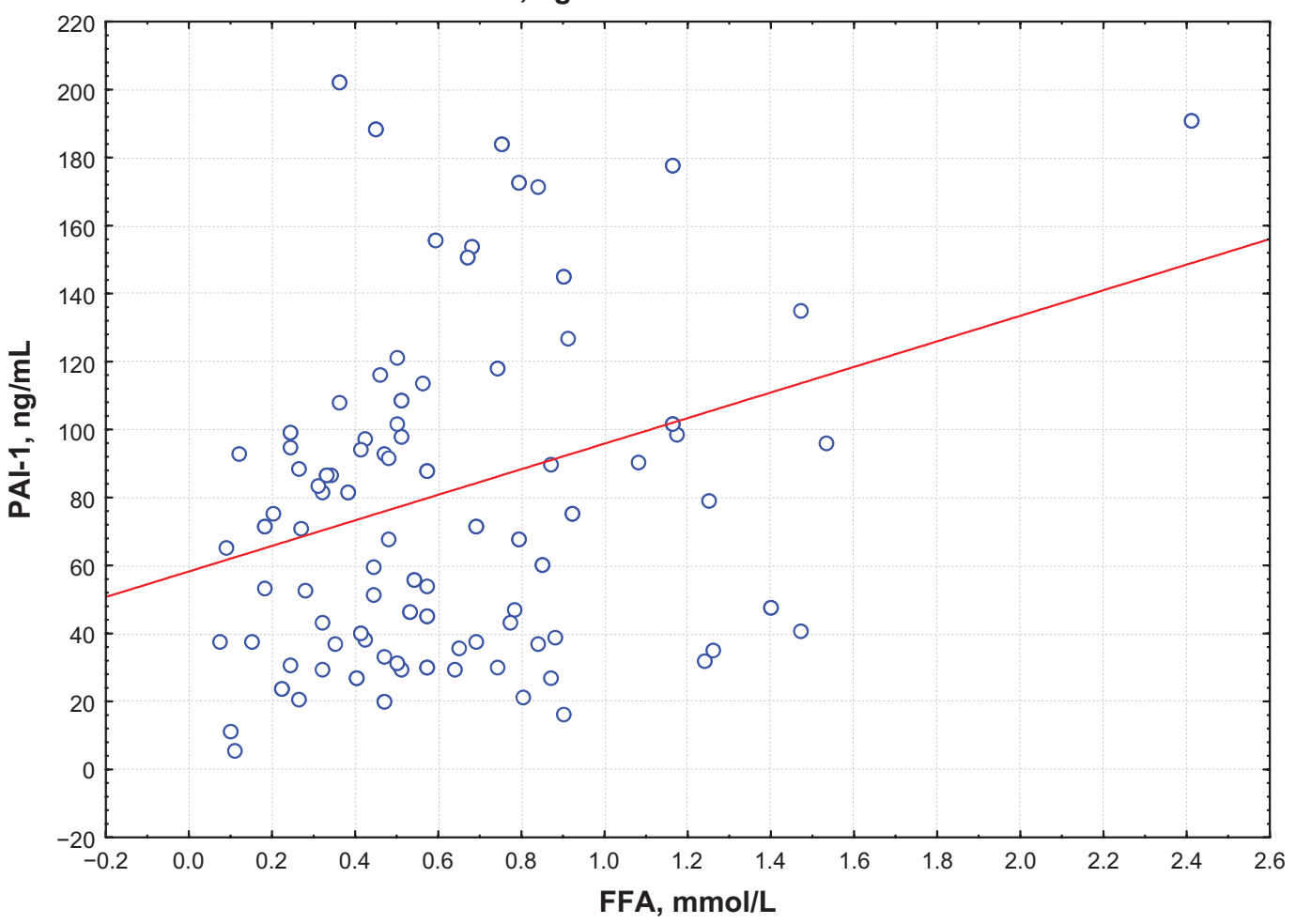

Figure 4 Correlation between FFA and PAI levels.

Abbreviations: FFA, free fatty acid; PAI-1, plasminogen activator inhibitor-1. 
evident in patients with a history of diabetes. FFA concentration remained high in the early recovery period after MI. Resistance to the antilipolytic effect of insulin in adipocytes may be one of the causes for the elevation of FFA concentration in MI patients. ${ }^{9}$ First, FFA becomes a substrate for triglyceride and atherogenic lipoprotein formation. Second, it prevents insulin binding to hepatocytes, increasing susceptibility to IR. Excessive FFA buildup in the liver leads to their abnormal metabolism. As a result, lipid metabolites (ceramides, diacylglycerides, and triacylglycerides) accumulate in hepatocytes causing the impairment of the insulin signaling pathway and, thus, the glucose transport to the cells and, as a consequence, its increase in blood. ${ }^{9}$ Altering hepatocytes by IR leads to a decrease in glycogen synthesis, activation of glycogenolysis, and gluconeogenesis enhancing hyperglycemia.

Metabolic abnormalities in myocardium cells under ischemic conditions are hypothesized to be an alternative mechanism resulting in blood FFA elevation during MI progression. FFAs are traditionally recognized as a main metabolic resource for myocardium. FFA oxidation provides two-thirds of the adenosine triphosphate (ATP) for the heart. The intensity of FFA transport to the heart cells is determined by their plasma concentration. Under myocardial ischemia conditions, FFA accumulates in blood due to the shift in biological oxidation to anaerobic glycolysis. ${ }^{9}$ The inhibition of insulin reception, accompanied by a decrease in the utilization of myocardium energy substrate and by accumulation of FFA in the blood, enhances the IR development, and is considered to be a negative lactacidosis effect. Moreover, the transport of fatty acids or ketone bodies into the heart and diaphragm determines the sharp drop in insulin sensitivity, according to obtained experimental data. ${ }^{9}$ Apparently, elevation of blood FFA levels in cases of acute myocardial infarction is associated not with myocardial ischemia alone, but also with the impaired insulin sensitivity in peripheral tissues, which is a result of an abundance of FFA in circulation. A direct positive correlation between postprandial insulin level and FFA concentration and a negative correlation between the QUICKI index and FFA level verified this hypothesis.

Regarding classical ideas, coronary artery thrombosis and plaque formation are the causes of MI occurrence. Thrombus formation is regulated by the fibrinolytic system, which prevents luminal occlusion. According to the results of recent studies, the enhanced activity of a key component of the fibrinolysis system, PAI-1, is considered not as a risk factor of atherosclerosis and MI only, but also as an IR marker. ${ }^{1,3}$ Moreover, previous experimental studies demonstrated that proinflammatory factors (interleukin-6 and CRP) could induce PAI-1 production. PAI-1 activity increase is regarded both as a risk factor for coronary atherothrombosis and MI and as a trigger for IR development. Alessi et al investigated hyperinsulinemic obese patients with the genotype associated with increased PAI-1 transcription, confirming a high risk of $\mathrm{MI}$ development. ${ }^{3}$ T2DM obese patients had elevated PAI-1 levels; however, PAI-1 level reduction was detected in this group after the administration of drugs improving insulin sensitivity in tissues. ${ }^{10}$ One of the explanations for this phenomenon was obtained in an observational study that suggested the PAI-1 role in the regulation of insulin reception. PAI-1 is able to block insulin signaling in adipocytes; exposure of adipocytes to high insulin concentrations was accompanied by increased PAI-1 expression in these cells. ${ }^{4}$ PAI-1 gene-knockout mice demonstrated the increased ability of insulin-stimulated glucose uptake compared to conventional mice. ${ }^{13}$ Our study reported increased PAI-1 concentration in both groups of patients; moreover, T2DM resulted in an increase of PAI-1 in the in-patient period. At the same time, there was a decrease in PAI-1 level in the early rehabilitation period after MI that led to a reduction of PAI-1 inhibiting influence and to normalization of fibrinolytic activity in both groups of patients. However, steadily high PAI-1 levels may indicate the risk of recurrent coronary events in patients with prior MI. Certain case-control studies found that high PAI-1 levels can be used to identify groups at high risk of CHD and T2DM. ${ }^{3}$ Therefore, first, an increase in PAI-1 level is associated with thrombophilia in MI patients. Second, PAI-1 level may help diagnose IR since there is negative correlation between PAI-1 level and IR occurrence that was verified by a low QUICKI index.

\section{Conclusion}

Thus, the results of this study show that MI progression is accompanied by insulin resistance development. The increase in FFA and PAI-1 concentrations is more informative regarding impaired sensitivity to insulin in MI as compared to postprandial glucose and insulin levels. High concentrations of FFA in MI patients seem to not only reflect the degree of myocardial ischemia but also to take part in hyperglycemia and IR development involving the sympathoadrenal system. The hyperactivation of the latter due to pain results in a more intensive lipolysis and excess FFA release. PAI-1 being the main regulator of the fibrinolytic system is also involved in the process of impaired insulin-receptor interaction and IR development in MI patients. Measuring metabolic markers of IR can be of high prognostic value for the risk stratification 
of acute coronary events and the choice of further treatment strategy. High levels of FFA and PAI-1 in MI still present in a stable patient's condition can be a theoretic rationale for the use of IR markers as one of the risk criteria for T2DM and as a solution to the issue of more effective secondary prevention.

\section{Disclosure}

The authors report no conflicts of interest in this work.

\section{References}

1. Collet JP, Montalescot G, Vicaut E, et al. Acute release of plasminogen activator inhibitor-1 in ST-segment elevation myocardial infarction predicts mortality. Circulation. 2003;108(4):391-394.

2. Ishihara M, Kojima S, Sakamoto T, et al; Japanese Acute Coronary Syndrome Study Investigators. Acute hyperglycemia is associated with adverse outcome after acute myocardial infarction in the coronary intervention era. Am Heart J. 2005;150(4):814-820.

3. Alessi MC, Juhan-Vague I. PAI-1 and the metabolic syndrome: links, causes, and consequences. Arterioscler Thromb Vasc Biol. 2006;26(10): 2200-2207.

4. López-Alemany R, Redondo JM, Nagamine Y, Muñoz-Cánoves P. Plasminogen activator inhibitor type-1 inhibits insulin signaling by competing with alphavbeta3 integrin for vitronectin binding. Eur J Biochem. 2003;270(5):814-821.

5. Juhan-Vague I, Morange PE, Frere C, et al; HIFMECH Study Group. The plasminogen activator inhibitor-1 -675 4G/5G genotype influences the risk of myocardial infarction associated with elevated plasma proinsulin and insulin concentrations in men from Europe: the HIFMECH study. $J$ Thromb Haemost. 2003;1(11):2322-2329.
6. Cefalu WT. Insulin resistance: cellular and clinical concepts. Exp Biol Med (Maywood). 2001;226(1):13-26.

7. Huxley R, Barzi F, Woodward M. Excess risk of fatal coronary heart disease associated with diabetes in men and women: meta-analysis of 37 prospective cohort studies. BMJ. 2006;332(7533):73-78.

8. Leclercq IA, Da Silva Morais A, Schroyen B, Van Hul N, Geerts A. Insulin resistance in hepatocytes and sinusoidal liver cells: mechanisms and consequences. J Hepatol. 2007;47(1):142-156.

9. Lopaschuk GD, Ussher JR, Folmes CD, Jaswal JS, Stanley WC. Myocardial fatty acid metabolism in health and disease. Physiol Rev. 2010;90(1):207-258.

10. Oliver MF. Sudden cardiac death: the lost fatty acid hypothesis. QJM. 2006;99(10):701-709.

11. Stanley WC, Recchia FA, Lopaschuk GD. Myocardial substrate metabolism in the normal and failing heart. Physiol Rev. 2005;85(3): 1093-1129.

12. Kryzhanovsky VA. Diagnosis and treatment of myocardial infarction. K: Phoenix; 2001.

13. Campbell IW. Antidiabetic drugs present and future: will improving insulin resistance benefit cardiovascular risk in type 2 diabetes mellitus? Drugs. 2000;60(5):1017-1028.

14. Liang X, Kanjanabuch T, Mao SL, et al. Plasminogen activator inhibitor-1 modulates adipocyte differentiation. Am J Physiol Endocrinol Metab. 2006;290(1):E103-E113.

15. Katz A, Nambi SS, Mather K, et al. Quantitative insulin sensitivity check index: a simple, accurate method for assessing insulin sensitivity in humans. J Clin Endocrinol Metab. 2000;85(7):2402-2410.

\section{Publish your work in this journal}

Diabetes, Metabolic Syndrome and Obesity: Targets and Therapy is an international, peer-reviewed open-access journal committed to the rapid publication of the latest laboratory and clinical findings in the fields of diabetes, metabolic syndrome and obesity research. Original research, review, case reports, hypothesis formation, expert opinion and commentaries are all considered for publication. The manuscript management system is completely online and includes a very quick and fair peer-review system, which is all easy to use. Visit http://www.dovepress.com/testimonials.php to read real quotes from published authors. 